\title{
Application of Artificial Neural Networks in Prediction of Compressive Strength of Concrete by Using Ultrasonic Pulse Velocities
}

\author{
Mr. P.S.Lande ${ }^{1}$, Mr. A.S.Gadewar ${ }^{2}$ \\ ${ }^{1}$ Associate Professor, Applied Mechanics Department, Government College of Engineering, Amravati, India \\ ${ }^{2}$ PG Student, Applied Mechanics Department, Government College of Engineering, Amravati, India
}

\begin{abstract}
As widely known, concrete is an essential material in civil engineering. However, its properties can vary considerably, depending on the nature and proportions of its constituents, the construction methods applied to create it, and the loading and environmental conditions to which it will be subjected over time. Therefore, the development of control methods to determine the condition and ascertain the quality of concrete is critical. Ultrasonic methods can play an important role in this area, since they allow us to monitor the density and homogeneity of concrete, providing information about the strength development and the existence of internal flaws and defects. To ensure that the information provided is reliable, expert knowledge is needed for interpreting the ultrasonic data. Computational tools that support the interpretation of ultrasonic (and other NDT) data might facilitate the job, reducing bias and helping specialists to analyze, in a consistent way, the great amount of data generated by the test. In this context, the use of Artificial Neural Networks (ANN) techniques is seen as a viable and adequate strategy to develop such tools. This study is focused on the evaluation of the feasibility of developing a specialist ANN tool. This kind of technique allows the emulation of the processes of specialists when dealing with uncertainty. Using a neural model, it is Possible to establish a non-linear correlation between known input data, such as ultrasonic readings and a certain output in this case, compressive strength, because this is the most used parameter to determine concrete quality.
\end{abstract}

Keywords: Compressive strength, Non-destructive testing, Ultrasonic pulse velocity, Regression analysis, Artificial neural network.

\section{Introduction}

The subject of using non-destructive testing (NDT) methods has received growing attention during recent years; especially during the rising need for quality characterization of damaged constructions made of concrete. The advantages of nondestructive tests as reduction in the labor consumption of testing, a decrease in labor consumption of preparatory work, a smaller amount of structural damage, a possibility of testing concrete strength in structures, where cores cannot be drilled and application of less expensive testing equipment as compared to core testing. These advantages are of no value if the results are not reliable, representative, and as close as possible to the actual strength of the structure. For finding in-situ concrete strength there are several destructive and non-destructive methods. Ultra Pulse Velocity testing is one of the most popular nondestructive techniques used in the assessment of the concrete properties in structures. The interpretation of the test results, however, is very difficult since UPV values are influenced by a number of factors although the UPV test is fairly simple and easy to apply.

The complexity of Concrete mixture makes the behavior of ultrasonic waves in concrete highly irregular, which, in turn hinders nondestructive testing. In the view of the problem complexities it would appear extremely optimistic to formulate an ultrasonic test method for the concrete strength determination. However, considering the seriousness of the infrastructure problem and the magnitude of the cost of rehabilitation, major advancement is desperately needed to improve the current situation. An unknown concrete mixture ratio in existing reinforced concrete structures is one of the most frequent issues that cause difficulties to determine the concrete compressive strength-UPV relationship. In this respect, the strength of concrete cannot be determined appropriately caused by the non-general pattern in the variability in the concrete mixture ratio findings obtained from laboratory researches. Thus, these findings cannot represent a general pattern for analysis as well. It is the main purpose of this study; an effective approach is presented by considering the compressive strength and UPV relationship of concrete samples. An Artificial Neural Network (ANN) approach for the estimation of the compressive strength of concrete specimens, using UPV and density values, is utilized in the study. Prediction of concrete compressive strength is implemented using ANN models, consisting of one input layer, one hidden layer and one output layer, for each data set. The analysis is then conducted for cube specimens with different compressive strengths for wide variation in their UPV and density. 
Application Of Artificial Neural Networks In Prediction Of Compressive Strength Of Concrete By

In previous studies, many approaches were presented by considering the compressive strength and UPV relationship of concrete samples. Hisham Y. Qasrawi ${ }^{1}$ presented study indicate that ANNs have strong potential as a feasible tool for predicting the compressive strength of concrete. Serkan Tapkin et-al ${ }^{2}$ proposed a neural network approach for the evaluation of concrete compressive strength by the use of ultrasonic pulse velocity values and some other factors. The neural network toolbox of MATLAB has been utilized in order to estimate the compressive strength of concrete specimens. Mürsel Erdal ${ }^{3}$ compare regression equations and an artificial neural network (ANN) developed for the estimation of compressive strength of vacuum processed concrete. Manish A. Kewalramani, Rajiv Gupta ${ }^{4}$ discuses for prediction of compressive strength of concrete based on weight and UPV for two different concrete mixtures. The prediction is done using multiple regression analysis and artificial neural depicts that artificial neural networks can be used to predict the compressive strength of concrete more effectively. J. Noorzaei ${ }^{5}$ focuses on development of Artificial Neural Networks (ANNs) in prediction of compressive strength of concrete after 28 days. A. Lorenzi ${ }^{6}$ focused on the evaluation of the feasibility of developing a specialist ANN tool to find concrete strength using ultra pulse velocity test. Using a neural model, the estimation power of the neural network is better than using traditional modeling techniques, such as regression analysis. Jerzy Hola, Krzysztof Schabowicz ${ }^{7}$ present new technique of nondestructively assessing the compression strength of concrete, which employs artificial neural networks.

\section{Non-destructive testing (NDT) of concrete using Ultrasonic Pulse Velocity(UPV)}

The Ultra Pulse Velocity (UPV) technique is one of the most popular non-destructive testing used in the assessment of concrete properties. Nevertheless, it is very difficult to accurately evaluate the concrete compressive strength with this method since UPV values are affected by a number of factors; which do not necessarily influence the concrete compressive strength to the some extent. Besides that the ultrasonic pulse velocity tester is the most commonly used one in practice. The UPV test is described in IS 13311(Part 1):1992 in detail.

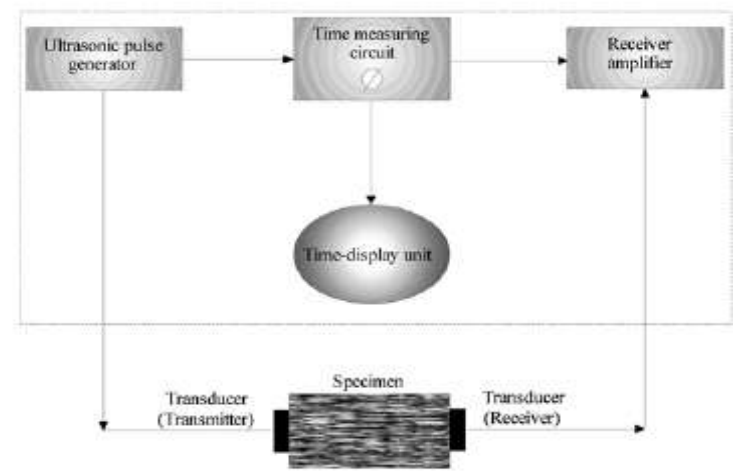

Fig.1 Schematic diagram of UPV testing circuit

Equipment shown schematically in Fig. 1, is actually used to determine the UPV through a known thickness of concrete. The ultrasonic pulse is created by applying a rapid change of potential from a transmitterdriver to a piezoelectric transformation element that causes it to vibrate at its fundamental frequency. The transducer is placed in contact with the material so that the vibrations are transferred to the material. The vibrations travel through the material and are then picked up by the receiver. The wave velocity is calculated using the time taken by the pulse to travel the measured distance between the transmitter and the receiver. If only very rough concrete surface is available for use, it is then required to smoothen and level the surface where the transducer is to be placed. The transducers are held tight on the surfaces of the specimens; and the display indicates the time of travel of the ultrasonic wave. This is a very convenient technique for evaluating concrete quality since the pulse velocity depends only on the elastic properties of the material and not on the geometry.

The longitudinal waves travel faster than the transverse waves. For this reason, the longitudinal waves are called primary $(\mathrm{P})$ waves and the transverse waves are called secondary $(\mathrm{S})$ waves. The dynamic modulus of elasticity of a homogenous and isotropic material can be determined by measuring the $\mathrm{P}$ and $\mathrm{S}$ wave velocities. The compression wave velocity can be expressed in terms of dynamic modulus of elasticity Ed and Poisson's ratio $v$ as follows

$$
V p=\sqrt{\frac{E d(1-v)}{\rho(1-2 v)(1+v)}}
$$

where $\rho$ is density of material; $V_{P}$ is primary wave velocity of the material. $V_{P}$ primary longitudinal wave velocity of material is determined in this study. The time the pulses take to travel through concrete is recorded in the test and subsequently the velocity is calculated as 


$$
V p=\frac{L}{T}
$$

where $V_{P}$ is the pulse velocity $(\mathrm{m} / \mathrm{s}), \mathrm{L}$ is the length $(\mathrm{m})$, and $\mathrm{T}$ is the effective time (s).

\section{Methods for estimating compressive strength of concrete}

Though the Non-Destructive Testing methods are relatively simple to perform, but the analysis for the interpretation of the test results are not so easy. Manufacturers of devices usually give empirical relationships for their own testing system. Such relationships are not suitable for every kind of concrete. Therefore, they need to be calibrated for the situation. For that special knowledge and the experience is required to analyze the properties of harden concrete. Here an attempt is made to interpret the test results of Ultrasonic pulse velocity for estimating compressive strength of unknown concrete mix.

a)Regression Analysis:

Regression Analysis is a statistical tool for the investigation of relationship between variable. Usually, the investigator seeks to ascertain the casual effect of one variable upon another. To explore such issues, the investigator assembles data on the underlying variables of interest and employs regression to estimate the quantitative effect of the casual variables upon the variable that they influence. In many ways regression analysis can be done depending on the need of accuracy which are given below

- Simple linear regression analysis

- Simple non-linear regression analysis

- Non- linear regression analysis

- Multiple regression analysis

A regression model assumes straight line relation between dependent and independent variable. By consensus, the accuracy of estimation of compressive strength of test specimens cast, cured, and tested under laboratory conditions by a properly calibrated devices lies between \pm 15 to $\pm 20 \%$. In order to compare the performance of existing single variable equations database determined in this study is employed. To compare prediction performances of existing equations root mean square error (RMSE) and coefficient of determination $\mathrm{R}^{2}$ are used. Equation $3 \& 4$ presents the calculation of RMSE \& $\mathrm{R}^{2}$ respectively.

$$
\begin{aligned}
& \left.R M S E=\sqrt{\frac{1}{n} \sum_{n}^{1}\left(f_{\text {c est }}\right.}-f_{\text {c mea }}\right)^{2} \\
& R^{2}=1-\frac{S S_{\text {err }}}{S S_{\text {tot }}}
\end{aligned}
$$

b) Artificial Neural Network

Artificial neural networks are non-linear data driven self adaptive approach. They are powerful tool for modeling, especially when the underlying data relationship is unknown. Therefore Artificial Neural Network, classed as Artificial Intelligence can use as computational tool for this purpose. Suitable neural network for the analysis of experimental results can be selected on the basis of literature on this subject.

An artificial neural network can be represented as a simplified model of the nervous system consisting of a large number of information processing elements. The elements are called artificial neurons. The prototype of the artificial neuron is the biological neuron. In its cybernetic counterpart the neuron's body is referred to as a processor. From the nerve cell originate thin, branching dendrites, constituting its "input" and a long thicker axon. In the artificial neuron this corresponds to input and output signal. The junction between two nerve cells called a synapse. Through the later signals are transmitted to other nerve cells. Information is transferred in the form of electric impulses called potentials. The structure of the nerve cell and that of its cybernetic counterpart are as shown in fig. 2

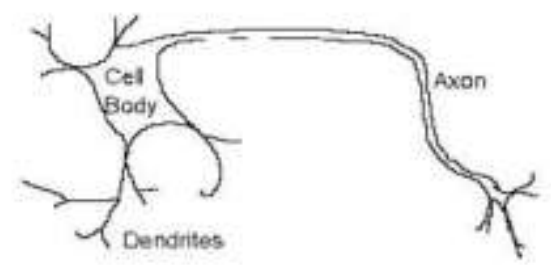

b)

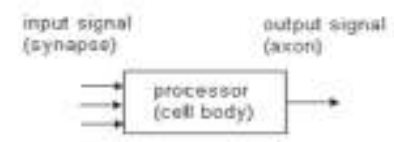

Fig.2 General structure of nerve cell (a) and its cybernetic counterpart (b) 
Artificial neurons connected together form a network. The structure of artificial neural networks is, as a rule, layered. Three functional groups can be distinguished in the artificial neural network, i.e. the inputs receiving signals from the networks outside and introducing them into its inside, the neurons which process information and the neurons which generate results.

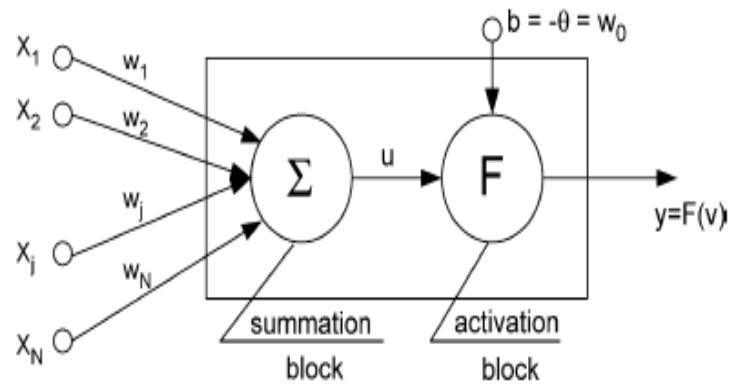

Fig. 3 Model of artificial neuron

A model of the artificial neuron is shown in Fig. 3 The model includes $\mathrm{N}$ inputs, one output, a summation block and an activation block. The following variables and parameters were used to describe the model shown in the figure above are

$X_{I}=\left(X_{1}, X_{2}, \ldots \ldots, X_{N}\right)$ an input vector,

$w_{I}=\left(w_{1}, w_{2}, \ldots \ldots, w_{N}\right)$ a weight vector,

$\mathrm{b}=-\square=w_{\mathrm{b}} \quad$ a bias

$v=u+b=\sum_{j=1}^{N} \mathrm{w}_{\mathrm{j}} \mathrm{x}_{\mathrm{j}}-\square$

Where

$$
\begin{array}{ll}
\sum_{j=1}^{N} \mathrm{w}_{\mathrm{j}} \mathrm{x}_{\mathrm{j}} & \text { a network potential } \\
\mathrm{F}(\mathrm{v}) & \text { an activation function }
\end{array}
$$

Characteristics of neural network:

1) The Neural networks exhibit mapping capabilities, i.e they can map input pattern to their associated output pattern.

2) The Neural networks learn by example, i.e. Neural network architectures can be 'trained' with known examples before they are tested for the 'inference'.

3) The Neural networks are robust system and are fault tolerant. Therefore it recall full pattern from incomplete, partial or noisy pattern.

4) The Neural networks can process information in parallel at high speed and in a distributed manner.

Depending on the way in which the neurons are connected, three types of artificial neural networks are distinguished: unidirectional networks, cellular networks and recursive networks

Development of an ANN Model:

The various steps in developing an neural network model are-

1) Variable selection: - The input variables important for modeling. Variables under study are selected by suitable variable selection procedures.

2) Formation of training, testing and validation sets:-

The data set is divided into three distinct sets called training, testing and validation sets. The training set is the largest set and is used by neural network to learn patterns present in the data. The testing set is used to evaluate the generalization ability of supposly trained network and final check on the performance of trained network is made using validation set.

3) Neural network architecture: - Neural network architecture defines its structure including number of hidden layers, number of hidden nodes and number of output nodes and activation function.

4) Evaluation criteria:-The most common error function minimized in neural networks is the mean squared errors.

5) Neural network training:-The objective of training is to find the set of weights between the neurons that determine the global minimum of error function. This involves decision regarding the number of iteration, selection of learning rate ( a constant of proportionality which determines the size of the weight adjustments made at each iteration) and momentum values ( how past weight changes affect current weight changes. 
Application Of Artificial Neural Networks In Prediction Of Compressive Strength Of Concrete By

\section{Methodology of the work}

Methodology to be adopted for strength identification by means of neural network on the basis of NDT are given below

1) Prepare data set for neural network from destructive and nondestructive tests.

2) Develop neural network which includes normalization and denormalization of data using MATLAB- N N

Toolbox, Selection of neural network, training and testing of selected network.

3) Validation of neural network.

\section{Experimental Study}

A total of 216 concrete cube of size $15 \times 15 \times 15 \mathrm{~cm}$ samples of unknown mix are tested using Ultrasonic Pulse Velocity tester for the determination of the direct ultra-pulse velocity $\left(\mathrm{V}_{\mathrm{d}}\right)$ before the execution of destructive compressive test The values of the $V_{d}$ are observed to be lying within $2.16 \mathrm{~km} / \mathrm{s}$ and $5.37 \mathrm{~km} / \mathrm{s}$; and the concrete cube compressive strengths varied between $2.23 \mathrm{MPa}$ and $40 \mathrm{MPa}$.

4.1 Regression Technique:

The goal of regression method is to fit a line through the points (results) such that the squared deviation of observed points from that line are minimize. Using Curve Fitting Toolbox of MATLAB (R2011a) different curve fitting models have been taken for optimizing the correlations.

For establishing correlation, plot has been generated taking UPV on X-axis and Compressive strength on Y-axis. Different linear and nonlinear models are tested and comparing their goodness of fit best correlation has been obtained as Eq.4 for which values of RMSE \& $\mathrm{R}^{2}$ are 0.00011 and 0.8766 respectively and Fig 6 shows Best fit results with 95\% confidence bound

$\mathrm{f}_{\mathrm{c}}=-1.067 \mathrm{E} 5 * \mathrm{~V}^{4}+2.374 \mathrm{E}^{4} * \mathrm{~V}^{3}-1868 * \mathrm{~V}^{2}+62.71 * \mathrm{~V}-0.7492$

where, $f_{c}=$ Compressive Strength of concrete $(\mathrm{MPa})$

$V=$ Direct Ultra-Pulse velocity $(\mathrm{km} / \mathrm{sec})$

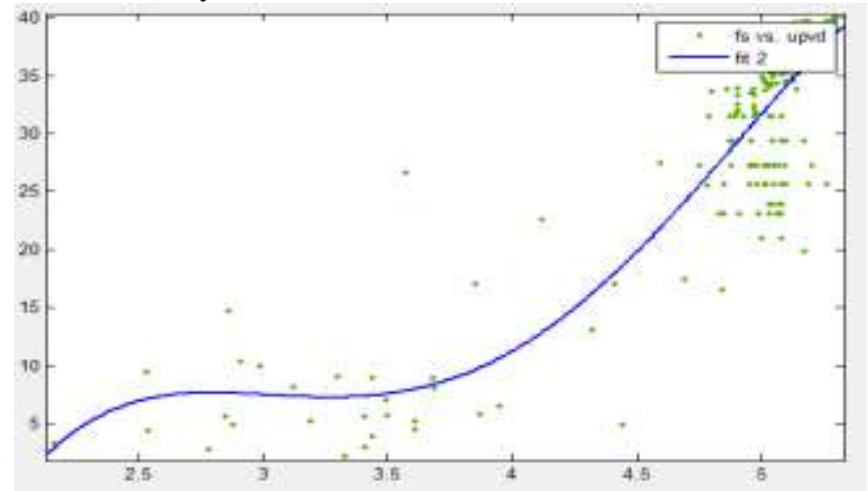

Fig.6 Best fit results with $95 \%$ confidence bound

\subsection{Artificial Neural Network:}

The problem can be defined as a nonlinear input-output relation among the factors UPV, and compressive strength of concrete values for ANN analyses. The typical multi-layer feed-forward ANNs consist of an input layer, one hidden layer and an output layer. This type of ANNs is used in the current application.

On the basis of a review of the literature the following feed forward error back propagation networks are selected

- The network with momentum and the descent gradient (GDM)

- The network descent gradient with adaptive learning rate (GDA)

- The Levenberg-Marquardt network (LM)

It should be noted that each of the above networks was trained \& tested for all the samples to find out the best one for the task.

The experimental data is saved in a computer file which is then used as input data for the network. The data was randomly divided into training $(80 \%)$, testing $(10 \%)$ and validation $(10 \%)$. They were normalizing by applying the procedure of the MATLAB-Neural Network Toolbox simulator to get MSE value nearer to zero and correlation coefficient $\mathrm{R}$ nearer to one. While training the network optimum numbers of neurons in the hidden layer and learning rate were calculated. The neural network learnt to identify the compressive strength of concrete cube samples. The training phase is stopped when the variation of error became sufficiently small. The ANN model is then tested and the results are compared by means of root mean squared error and coefficient of determination. 
Table 4.1. Optimum elements of different network architecture

\begin{tabular}{|c|c|c|c|c|}
\hline $\begin{array}{l}\text { Sr. } \\
\text { No. }\end{array}$ & NDT & $\begin{array}{c}\text { Short } \\
\text { Name of } \\
\text { Neural } \\
\text { Network }\end{array}$ & $\begin{array}{l}\text { Neurons } \\
\text { in } \\
\text { Hidden } \\
\text { Layer }\end{array}$ & $\begin{array}{l}\text { Learn } \\
\text {-ing } \\
\text { Rate }\end{array}$ \\
\hline 1 & \multirow{3}{*}{$\begin{array}{l}\text { Ultrasonic } \\
\text { Pulse } \\
\text { Velocity }\end{array}$} & GDM & 20 & 0.1 \\
\hline 2 & & GDA & 40 & 0.2 \\
\hline 3 & & LM & 10 & 0.02 \\
\hline
\end{tabular}

Table4.2. The performance different network architecture

\begin{tabular}{|c|c|c|c|c|}
\hline $\begin{array}{l}\text { Sr. } \\
\text { No. }\end{array}$ & NDT & $\begin{array}{c}\text { Short } \\
\text { Name of } \\
\text { Neural } \\
\text { Network }\end{array}$ & $\begin{array}{l}\text { Mean } \\
\text { Sq. } \\
\text { Error }\end{array}$ & $\mathrm{R}$ \\
\hline 1 & \multirow{3}{*}{$\begin{array}{c}\text { Ultrasonic } \\
\text { Pulse } \\
\text { Velocity }\end{array}$} & GDM & $3.79 \mathrm{E}-4$ & 0.95 \\
\hline 2 & & GDA & $4.95 \mathrm{E}-4$ & 0.999 \\
\hline 3 & & LM & $5.99 \mathrm{E}-5$ & 0.968 \\
\hline
\end{tabular}

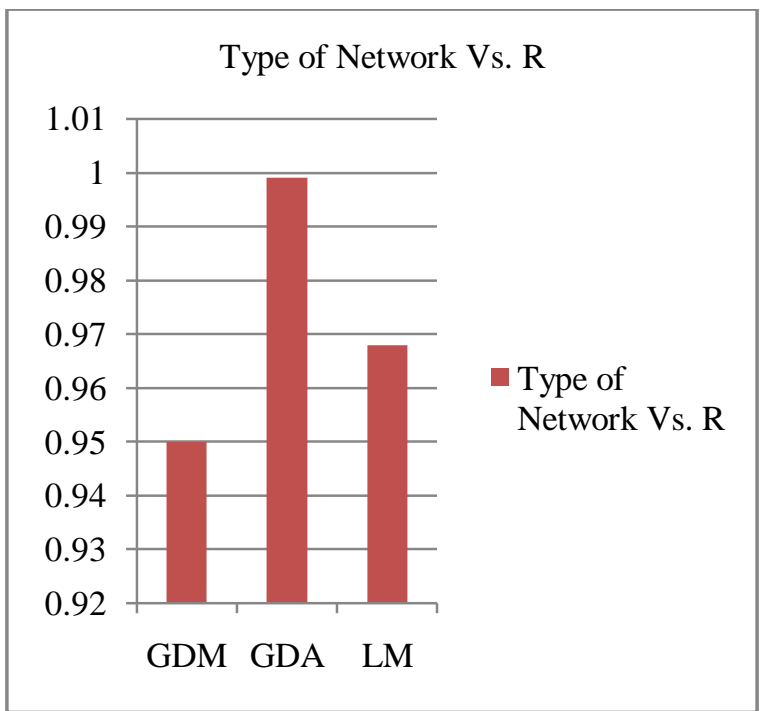

Fig.6 The values of $\mathrm{R}$ for different network architecture

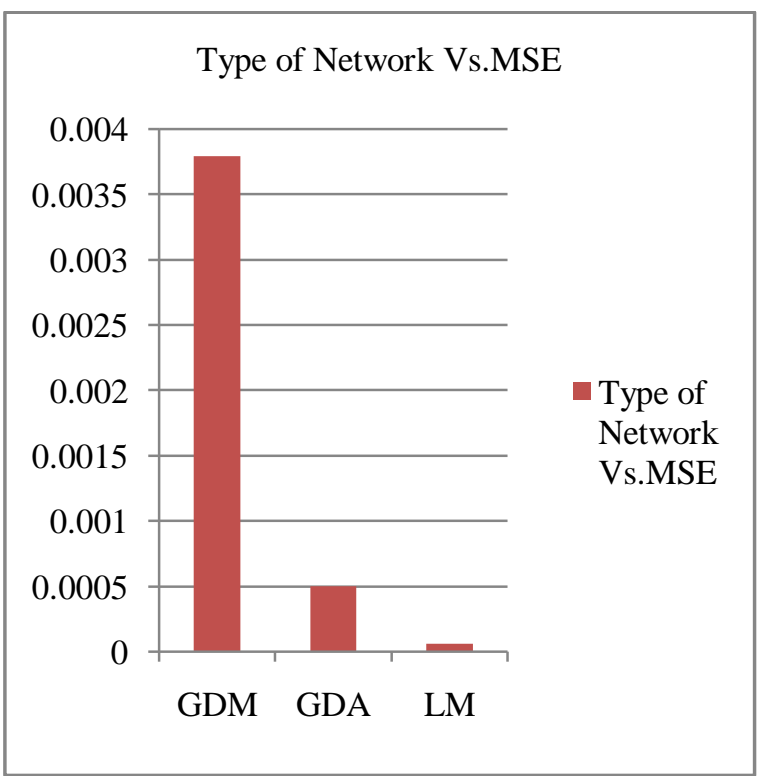

Fig.7 The values of MSE for different network architecture 
Finally the Levenberg-Marquardt network (LM) was selected on the basis low training and testing MSE value as well as high correlation coefficient $\mathrm{R}$ with low learning rate, the obtained result was give in the table 4.1 \& 4.2 .

\section{Result and Discussion:}

The testing set is employed to evaluate the confidence in the performance of the trained network. MSE and $\mathrm{R}$ values for different neural networks are shown in Fig. 6 \& 7. The Levenberg-Marquardt network (LM) was selected on the basis low training and testing MSE value and high correlation coefficient $\mathrm{R}$ with low learning rate, the obtained result is given in the table $4.1 \& 4.2$.

After carrying out number of trainings in the neural network simulation, the optimum hidden neuron number and hidden layer number are determined as 10 and 1, respectively and parameters for LevenbergMarquardt network (LM) found as Learning rate 0.02 with training performance goal $10^{-5}$, momentum constant 1.0 and maximum number of epochs 1000 with activation functions as tangent sigmoid. It can be seen that the smallest MSE and the highest R values are obtained by 10 hidden neurons in hidden layer. The analyst had the optimum flexibility to be able to determine the number of hidden neuron numbers, on a MSE basis. Table 5.3. shows the performance of the LM network for different hidden neuron numbers

Table 4.3. The performance of the LM network for different hidden neuron numbers

\begin{tabular}{|c|c|c|c|c|}
\hline $\begin{array}{l}\text { Sr. } \\
\text { No. }\end{array}$ & $\begin{array}{c}\text { Neurons } \\
\text { in } \\
\text { Hidden } \\
\text { Layer }\end{array}$ & $\begin{array}{c}\text { Learn } \\
\text {-ing } \\
\text { Rate }\end{array}$ & $\begin{array}{c}\text { Mean } \\
\text { Square } \\
\text { Error } \\
\text { MSE }\end{array}$ & $\begin{array}{c}\text { Correlation } \\
\text { Coefficient } \\
\text { R }\end{array}$ \\
\hline 1 & 10 & 0.02 & $\begin{array}{l}5.99 \\
\text { E-05 } \\
\end{array}$ & 0.967 \\
\hline 2 & 20 & 0.02 & $\begin{array}{l}1.25 \\
\text { E-04 }\end{array}$ & 0.948 \\
\hline 3 & 30 & 0.02 & $\begin{array}{l}1.32 \\
\text { E- } 04\end{array}$ & 0.951 \\
\hline 4 & 40 & 0.02 & $\begin{array}{l}5.72 \\
\text { E-05 }\end{array}$ & 0.935 \\
\hline 5 & 50 & 0.02 & $\begin{array}{l}1.94 \\
\text { E-04 }\end{array}$ & 0.943 \\
\hline 6 & 60 & 0.02 & $\begin{array}{l}3.11 \\
\text { E-04 }\end{array}$ & 0.923 \\
\hline 7 & 70 & 0.02 & $\begin{array}{l}2.17 \\
\text { E-04 }\end{array}$ & 0.924 \\
\hline
\end{tabular}

It can be seen that the model of hidden layer with 10 neurons has the smallest MSE (5.99E-5 MPa), and it has the highest $R(0.95733)$. The best validation performance is obtain at 1 epoch and MSE value is 5.99E-5 MPa shown in Fig.8.It is not surprising to observe some fluctuations in the mean squared errors due to the nature of the back propagation algorithm. However, it is observed that the modeling results are exceptionally close to the real compressive strength test results; therefore there is no doubt regarding the accuracy of the MSE values.

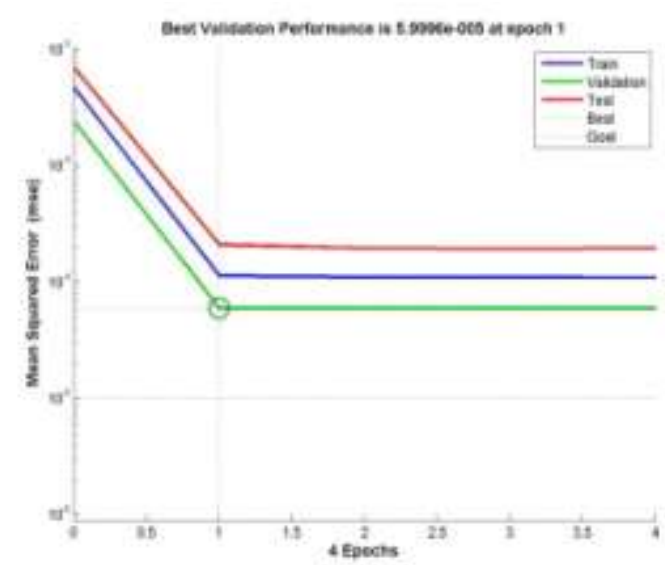

Fig.8 The performance of Levenberg-Marquardt network (LM) 


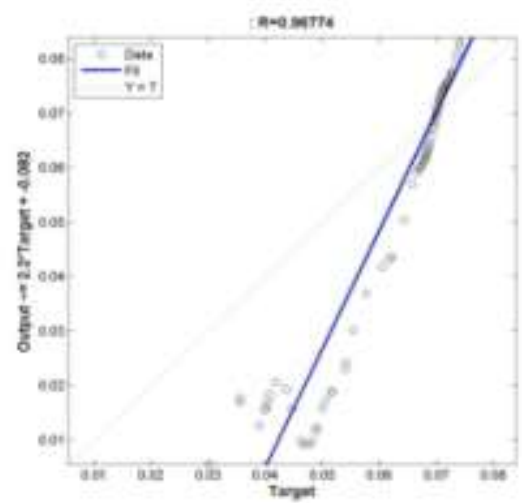

Fig.9The regression of Levenberg-Marquardt network (LM)

Table 4.4. Comparison of results using Regression and ANN

\begin{tabular}{|c|l|c|}
\hline \% Error & Regression & ANN \\
\hline Average & 4.67 & 1.77 \\
\hline Max. & 30.73 & 28.33 \\
\hline Min. & -58.18 & -46.21 \\
\hline
\end{tabular}

Table 4.4 clearly depicts the comparison of results using regression analysis and ANN in terms of average, maximum and minimum percentage of error involved in prediction of concrete compressive strength based UPV of concrete specimen. As seen the maximum percentage error through ANN is 28.33 whereas for same set of data points error through regression is 30.73. A better inference can be drawn from values of average percentage error as regression produces higher value of average percentage error than ANN prediction, that ANN prediction is observed to perform better for prediction of compressive strength.

The results are plotted in the form of a graph showing comparison of prediction through two methods of analysis i.e., regression analysis and ANN. Fig.10 shows predicted compressive strengths through regression analysis and ANN for A cubes. The figure clearly depicts that experimentally evaluated values of compressive strength are in strong coherence with the values predicted through ANN than regression analysis for most of the samples.

\section{Conclusions}

This study indicates the ability of the multilayer feed forward back propagation neural network as a good technique for model the concrete compressive strength and UPV relationship. The ANN model performs sufficiently in the estimation of concrete compressive strength. Gradient descent algorithm and one hidden layer are employed in the analysis. The MSE values are reasonably small indicating that the estimates are most accurate and the trained network yield superior results.

The neural network model to predict compressive strength based on UPV of concrete specimens is utilized in this study. The prediction made using ANN shows a high degree of consistency with experimentally evaluated compressive strength of concrete specimens used. Thus, the present study suggests an alternative approach of compressive

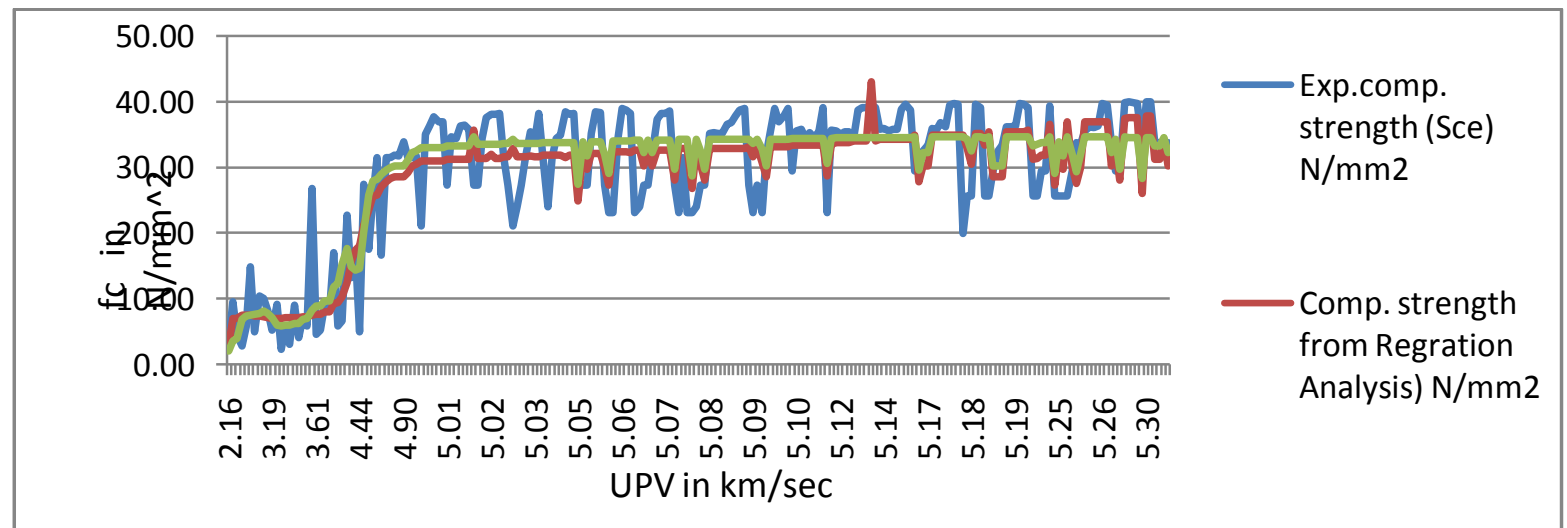

Fig. 10 Predicted compressive strengths through Regression and ANN for A cubes 
strength assessment against destructive testing methods.

This current study employed data set which is composed of limited input and output vectors. Therefore, it would be reasonable to propose a further works using more data sets from various areas could be needed to generalize the conclusions in this study.

\section{References}

[1] Qasrawi H. Y., 'Concrete strength by combined nondestructive methods simply and reliablityy', Cement and Concrete Research, 30 (Civil), 739-746, 2000.

[2] Tapkin S., Tucan M., Arioz O., Tucan A., 'Estimation of Concrete Compressive Strength by Using Ultrasonic Pulse Velocities and Artificial Neural Network', 1-7.

[3] Erdal M., 'Prediction of the compressive strength of vacuum processed concretes using artificial neural network and regression techniques', Scientific Research and Essay 4 (10), 1057-1065, 2009.

[4] Kewalramani M., Gupta R., 'Concrete compressive strength prediction using ultrasonic pulse velocity through artificial neural networks', Automation in Construction 15, 374 - 379, 2006.

[5] Noorzaei J., Hakim S., Jaafar M., Thanoon W., 'Development of Artificial Neural Network For Predicting Concrete Compressive Strength', International Journal of Engineering and Technology, 4( 2), 141-153, 2007.

[6] Hola J., Schabowicz K., 'Application of Artificial Neural Netwoks To Determine Concrete compressive Strength Based On Nondestructive Test', Journal of Civil Engineering And Management, XI (1), 23-32, 2005 